alpha-induced thyroid dysfunction in patients with chronic hepatitis C. Eur J Endocrinol 142:431-437

6. Cohen IR (2001) Antigenic mimicry, clonal selection and autoimmunity. J Autoimmun 16:337-340

7. Oldstone MBA (1998) Molecular mimicry and immune-mediated diseases. FASEB J 12:1255-1265

8. Weiner AJ, Geysen HM, Christopherson C et al (1992) Evidence for immune selection of hepatitis $\mathrm{C}$ virus (HCV) putative envelope glycoprotein variants: potential role in chronic HCV infections. Proc Natl Acad Sci USA 89:3468-3472

9. Christen U, Edelamn KH, McGavern DB et al (2004) A viral epitope that mimics a self antigen can accelerate but not initiate autoimmune diabetes. J Clin Invest 114:1290-1298

10. Chang KM, Gruener NH, Southwood S et al (1999) Identification of HLA-A3 and -B7-restricted CTL response to hepatitis $\mathrm{C}$ virus in patients with acute and chronic hepatitis $\mathrm{C}$. J Immunol 162:1156-1164

11. Carella C, Mazziotti G, Amato G, Breverman LE, Roti E (2004) Interferon-? related thyroid disease: pathophysiological, epidemiological and clinical aspects. J Clin Endocrinol Metab 89:3656-3661

Intern Emerg Med (2007) 2:67-70

DOI 10.1007/s11739-007-0019-9

\section{Transient massive hyperlipidaemia in a type 2 diabetic subject}

\section{G.B. Vigna • A. Passaro • K. Bonomo • G. Anfossi R. Fellin • M. Trovati}

G.B. Vigna $(\boldsymbol{凶}) \cdot$ A. Passaro $・$ R. Fellin Section of Internal Medicine, Gerontology and Geriatry Department of Clinical and Experimental Medicine University of Ferrara

Via Savonarola 9, I-44100 Ferrara, Italy e-mail:vgg@unife.it

K. Bonomo • G. Anfossi • M. Trovati Diabetes Unit Department of Clinical and Biological Sciences University of Turin San Luigi Gonzaga Hospital, Orbassano (Turin), Italy

Received: 7 April 2006 / Accepted in revised form: 13 September 2006 / Published online: 31 March 2007

A 50-year-old man, in apparently good health, was referred to the Lipid Center of San Luigi Gonzaga Hospital, Orbassano (Turin, Italy), by his primary care physician (PCP) because clinical tests at the time of blood donation showed milky plasma with serious hypertriglyceridaemia (>5000 mg/dl) and hyperglycaemia (381 mg/dl), diagnostic for diabetes. It was not possible to perform further blood chemical analyses because hypertriglyceridaemia would have provided abnormal results. The patient entered the hospital to prevent acute pancreatitis, which is often associated with severe hypertriglyceridaemia.

The clinical history revealed $H$. pylori-associated gas- tric ulcer in the prior year. At that time, laboratory tests disclosed "mild" hyperglycaemia and hypertriglyceridaemia (the patient's records were misplaced.) An ill-defined hypolipidaemic diet was recommended at discharge, but the suggestion was neglected. He was a former smoker (30 pack year, stopping about 10 years before), and consumed a diet rich in saturated fats and carbohydrates while alcohol ingestion was mild and occasional (2-3 drinks per week). His living mother was affected by type 2 diabetes; his father died by accident at a young age, while a brother suffered a myocardial infarction at age 43 , seemingly not related to traditional cardiovascular risk factors. The patient weighed $79 \mathrm{~kg}$ and was $1.75 \mathrm{~m}$ tall (Body Mass Index, $\mathrm{BMI}=25.8 \mathrm{~kg} / \mathrm{m}^{2}$ ), and there had been no significant recent weight modification. No significant abnormalities were detected at physical examination.

Biochemical analyses (Table 1) corroborated the findings of severe hypertriglyceridaemia $(6.594 \mathrm{mg} / \mathrm{dl})$, showing also high cholesterol levels $(658 \mathrm{mg} / \mathrm{dl})$ and low plasma highdensity lipoprotein cholesterol (HDL-C, $15 \mathrm{mg} / \mathrm{dl}$ ); apolipoprotein A-I was $75 \mathrm{mg} / \mathrm{dl}$ and apolipoprotein B was $141 \mathrm{mg} / \mathrm{dl}$, both within normal laboratory range. After refrigeration overnight at $+4^{\circ} \mathrm{C}$, serum showed a creamy surface layer and a turbid infranatant, while lipoprotein electrophoresis disclosed lipids at origin and a broad pre- $\beta$ band: both these tests indicated the presence of chylomicrons and very low-density lipoproteins (VLDLs). Plasma creatinine was within normal limits, while urinalysis detected trace amounts of glucose and ketone bodies; liver and thyroid functions were also normal; $\gamma$-glutamyl-transferase was slightly increased and serum sodium decreased. Glucose level $(215 \mathrm{mg} / \mathrm{dl})$ and glycated haemoglobin (HbA1c) were increased, C-peptide was in the normal-high range, while immune parameters were unaffected, in particular antiislet cells antibodies (ICA) and glutamic acid decarboxylase antibodies (GADA) were not detected.

Preparative ultracentrifugation was carried out, showing cholesterol enrichment in the density fraction $<1.006 \mathrm{~g} / \mathrm{ml}$, corresponding to chylomicrons+VLDLs. Apolipoprotein E was homozygous for the most common isoform $(\varepsilon 3 / \varepsilon 3$ genotype). We did not disclose a deficiency of apolipoprotein C-II, which represents the physiological lipoprotein lipase (LPL) activator, but we could not directly evaluate LPL activity.

Supra-aortic and lower limb echo-doppler examination revealed increased intima-media thickness but no plaques, while liver echotomography showed diffuse, high-grade steatosis.

Along with clinical evaluation, dietetic therapy and a four-injection insulin regimen was administered (3 short-acting pre-prandial insulin, and one bedtime long-acting dose). Treatment resulted in rapidly improving and rather steady glycaemic control with progressive reduction of insulin requirement. At the same time the dyslipidaemia was treated with low-fat diet, subcutaneous calcium heparin (2000 IU, 
Table 1 Biochemical tests after hospital admission

\begin{tabular}{llllll}
\hline Cholesterol & $658 \mathrm{mg} / \mathrm{dl}$ & Anti-insula Ab & Negative & Uric acid & $5.8 \mathrm{mg} / \mathrm{dl}$ \\
Triglycerides & $6594 \mathrm{mg} / \mathrm{dl}$ & Anti-GAD Ab & Negative & Total proteins & $7.8 \mathrm{~g} / \mathrm{dl}$ \\
HDL-chol & $15 \mathrm{mg} / \mathrm{dl}$ & Leucocytes & $4290 / \mathrm{mm} 3$ & ALT & $26 \mathrm{U} / \mathrm{l}$ \\
Apo AI & $75 \mathrm{mg} / \mathrm{dl}$ & Haemoglobin & $14.1 \mathrm{~g} / \mathrm{dl}$ & $\gamma$-Glutamyl-transferase & $112 \mathrm{U} / \mathrm{l}$ \\
Apo B & $141 \mathrm{mg} / \mathrm{dl}$ & hs-CRP & $0.26 \mathrm{mg} / \mathrm{dl}$ & Bilirubin & $0.5 \mathrm{mg} / \mathrm{dl}$ \\
Fasting glycaemia & $215 \mathrm{mg} / \mathrm{dl}$ & Sodium & $133 \mathrm{mEq} / \mathrm{l}$ & Amylase & $30 \mathrm{U} / \mathrm{L}$ \\
Glycated haemoglobin & $10.5 \%$ & Potassium & $4.5 \mathrm{mEq} / \mathrm{l}$ & TSH & $2.2 \mu \mathrm{UI} / \mathrm{ml}$ \\
C-peptide & $3.5 \mathrm{ng} / \mathrm{ml}$ & Creatinine & $0.9 \mathrm{mg} / \mathrm{dl}$ & f-Thyroxine & $1.2 \mathrm{ng} / \mathrm{dl}$ \\
\hline
\end{tabular}

bid), fenofibrate (200 mg, qd) and omega-3 fatty acids ( $1 \mathrm{~g}$, tid). Plasma triglycerides decreased in a few days, followed by blood cholesterol (Fig. 1). After lipid pattern improvement, insulin treatment was completely discontinued, and the patient was successfully treated with only diet and metformin; also subcutaneous heparin was stopped, while fibrates and polyenoic fatty acids were maintained. His weight decreased by 3-4 kg during the hospital stay, while 6 months afterwards his BMI was $25.2 \mathrm{~kg} / \mathrm{m}^{2}$.

In short, we report a case of a patient with massive dyslipidaemia associated with glycaemic decompensation, in the framework of previously unrecognised type 2 diabetes, anamnestic hints of "slight", not further defined, hyperglycaemia and a familial history of diabetes. Increased HbA1c plasma levels suggest that diabetic decompensation occurred during weeks or months before, and not in a few days time, while high C-peptide plasma levels in the context of overt hyperglycaemia testify to a relative insulin deficiency and marked insulin resistance. Finally, the absence of immune markers rule out the presence of latent autoimmune diabetes in the adult (LADA), a peculiar form

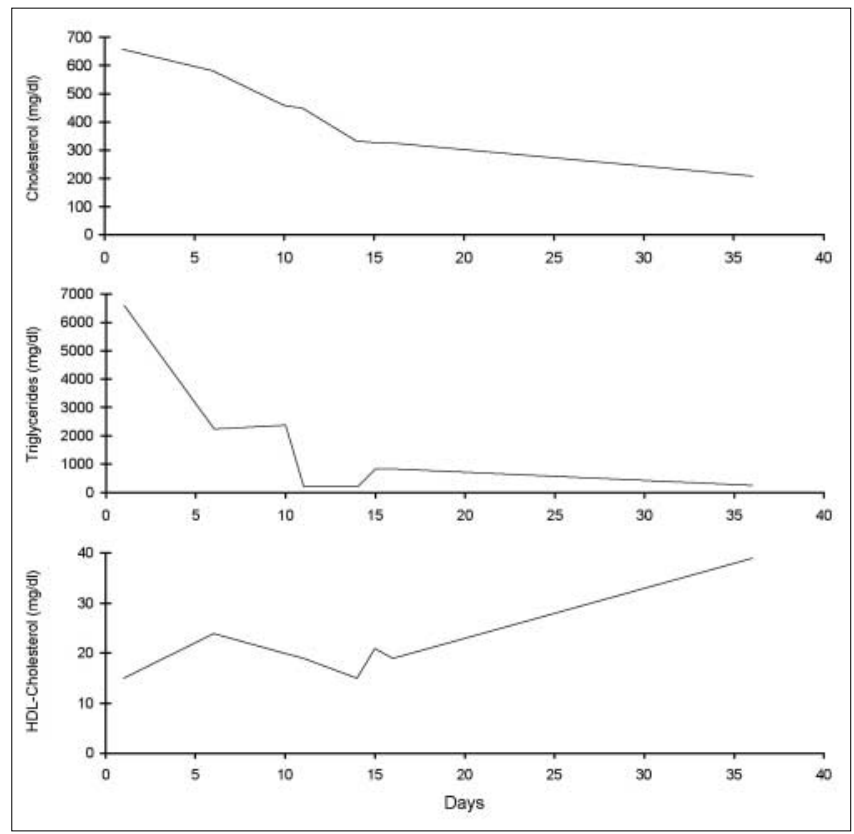

Fig. 1 Lipoprotein parameters during hospital stay and after discharge of smoldering type 1 diabetes that appears during adulthood. Dyslipidaemia is a common finding in diabetic populations, and represents a major coronary heart disease risk factor [1]. It may be found when type 2 diabetes is diagnosed, persisting in spite of hypoglycaemic therapy and closely related to insulin-resistance parameters. It is generally characterised by moderate hypertriglyceridaemia and low HDL-C plasma levels, while total and low-density lipoprotein-cholesterol (LDL-C) concentrations are similar to non-diabetic subjects; however LDL particles are smaller and denser than usual, and possibly more atherogenic. Moreover, the LDLs and HDLs are triglyceride-enriched [2,3]. Less frequently, diabetes begins with a chylomicronemic syndrome. In our patient, hyperlipidaemia was at first characterised by very high triglycerides and cholesterol plasma levels. Blood triglycerides exceeding $2000 \mathrm{mg} / \mathrm{dl}$ are a rare clinical finding; when such values are present, several haematochemical parameters may look abnormal as a result of analytical interference or uneven analyte distribution (the "false" hyponatraemia we found is ascribable to the presence of sodium only in the watery plasma phase, while the lipid phase, about $10 \%$ of total plasma volume, is not permeable to sodium).

The most common primary causes of severe hypertriglyceridaemia are familial LPL deficiency and familial apo C-II deficiency (the physiological LPL activator), which may account for up to $10 \%$ of all individuals referred to a lipid clinic with the chylomicronaemia syndrome [4]. The transient hypertriglyceridaemia of our patient was not in agreement with familial conditions, characterised by rather steadily increased lipid values (also above 5-10 000 $\mathrm{mg} / \mathrm{dl}$ ), and not responsive to drug treatment. The coexistence of high plasma cholesterol and triglycerides with fasting chylomicrons may suggest type III hyperlipidaemia. This lipid disorder (also known as 'dysbetalipoproteinaemia' or 'broad-beta disease') is a rare condition (1:10 000 subjects) and much more frequent in subjects with an uncommon apolipoprotein E genotype ( $\varepsilon 2 / \varepsilon 2)$. It is characterised by the development of premature atherosclerosis and plasma accumulation of partially catabolised chylomicrons and VLDLs [5]. Dyslipidaemic patients show increased cholesterol and triglyceride levels $(>300 \mathrm{mg} / \mathrm{dl}$ ) and a peculiar "broad beta" band between $\beta$ (LDL) and pre- $\beta$ (VLDL) bands at lipoprotein electrophoresis; chylomicrons may be present in variable amounts. In this case, 
however, a very distinct electrophoretic $\beta$-band and a common apolipoprotein $\varepsilon 3 / \varepsilon 3$ genotype rule out the hypothesis of type III hyperlipoproteinaemia.

Another rare hyperchylomicronaemic syndrome (with a prevalence of about 1:10 000 in the general population) is Fredrickson type V dyslipidaemia. In this condition both VLDL and chylomicron levels are increased, while plasma triglycerides are very high. It may display either a primary or a secondary origin: in the latter case obesity, pregnancy, ethanol intake, estro-progestins, $\beta$-blockers or anion exchange resins, hypothyroidism, kidney disease and diabetes may favour its appearance in predisposed individuals. Our patient seems classifiable in this type of dyslipidaemia. Likely some concurrent lipid metabolism abnormality (e.g., familial combined hyperlipidaemia or heterozygous LPL deficiency) may represent the fuel promoting hyperchylomicronaemia (+VLDLs) in response to hyperglycaemic decompensation, most probably feed-back by incoming hypertriglyceridaemia as part of a well known vicious cycle $[6,7]$. This fact might explain both transient type IIb hyperlipidaemia during clinical type $\mathrm{V}$ remission, and the familial history of coronary heart disease. His lipid profile 6 months after hospital discharge was alternatively normal, or suggestive of type IV hyperlipaemia. Our diagnosis is probable but the pathophysiology is presumptive, as important data are lacking (LPL activity or gene sequencing); however therapy response and patient followup add reliability to this interpretation.

Interestingly our patient was substantially asymptomatic: clinical complications of hyperchylomicronaemia (acute pancreatitis, eruptive xanthomatosis) are particularly common with plasma triglyceride levels above $2000 \mathrm{mg} / \mathrm{dl}$ [4], but sometimes, unpredictably, they may be absent. The relationship between hyperchylomicronaemia and premature atherosclerosis is still controversial, denied by most authors, but has received some valuable confirmation [8].

Treatment improved the lipid pattern after only a few days. A lipid-poor diet, in particular, represents the cornerstone of early treatment. Fibrates are then particularly indicated: after interacting with a specific nuclear receptor (peroxisome proliferator activated receptor- $\alpha$, PPAR $\alpha$ ), they fasten LPL-induced lipolysis (especially by blocking apo C-III synthesis, a main lipoprotein lipase-inhibitor), and decrease liver triglycerides and apolipoprotein B secretion [9]. Heparin was effective in the short term, but its role in severe hypertriglyceridaemia is still under discussion [10]. It has been defined as a "plasma clearing factor" showing hypotriglyceridaemic effect in an acute setting, and may be useful when pancreatitis supervenes, especially in conjunction with insulin treatment $[10,11]$. Heparin may release LPL from the endothelial surface of extrahepatic tissues, promoting its access to blood where the enzyme hydrolyses VLDL and chylomicron triglycerides. However, after a quick peak effect, chronic heparin infusion causes lipase activity to reach a plateau that is substantially lower than the former level (about
$15 \%$ ), decreasing correspondingly the tissue stores (likely by hepatic catabolism of circulating LPL molecules) [12]. This makes its long-term utilisation particularly questionable. Omega-3 fatty acids are another debated drug; they display hypotriglyceridaemic effect when administered to subjects with high VLDL plasma levels, while in type $\mathrm{V}$ patients there is no consensus on their efficacy (they could even be detrimental, favouring chylomicron synthesis in enterocytes). Also plasmapheresis may represent, in selected contexts, a useful but extreme therapeutical tool [13].

Finally, insulin effect must not be underestimated: it is always advisable in diabetic patients when marked hypertriglyceridaemia is recognised. In fact insulin can stimulate LPL production, inhibit endogenous triglyceride synthesis and promote their catabolism [14].

\section{References}

1. Syvanne M, Taskinen M-R (1997) Lipids and lipoproteins as coronary risk factors in non insulin dependent diabetes mellitus. Lancet 350[Suppl 1]:20-23

2. Fellin R, Vigna GB, Zuliani G (2002) Disordini del metabolismo delle lipoproteine. In: Crepaldi G (ed) Trattato di Medicina Interna, Vol I. Piccin, Padova, pp 1433-1461

3. Durrington P (2003) Dyslipidemia. Lancet 362:717-731

4. Brunzell JD, Deeb SS (2001) Familial lipoprotein lipase deficiency, apo C-II deficiency, and hepatic lipase deficiency. In Scriver CR et al (eds) The metabolic and molecular bases of inherited disease. McGraw Hill, New York, pp 2789-2816

5. Mahley RW, Rall SC Jr (2001) Type III hyperlipoproteinemia (dysbetalipoproteinemia): the role of apolipoprotein $\mathrm{E}$ in normal and abnormal lipid metabolism. In Scriver CR et al (eds) The metabolic and molecular bases of inherited disease. McGraw Hill, New York, pp 2835-2862

6. Yang T, Pang CP, Tsang MW et al (2003) Pathogenic mutations of the lipoprotein lipase gene in Chinese patients with hypertriglyceridemic type 2 diabetes. Hum Mutat 21:453

7. Mingrone G, Henriksen FL, Greco AV, Krogh LN, Capristo F, Gastaldelli A, Castagneto M, Ferrannini E, Gasbarrini G, Beck-Nielsen H (1999) Triglyceride-induced diabetes associated with familial lipoprotein lipase deficiency. Diabetes 48:1258-1263

8. Benlian P, De Gennes JL, Foubert L et al (1996) Premature atherosclerosis in patients with familial chylomicronemia caused by mutations in the lipoprotein lipase gene. $\mathrm{N}$ Engl J Med 335:848-854

9. Deprés JP, Lemieux I, Robins SJ (2004) Role of fibric acid derivatives in the management of risk factors for coronary heart disease. Drugs 64:2177-2198

10. Berger Z, Quera R, Poniachik J et al (2001) [Heparin and insulin treatment of acute pancreatitis caused by hypertriglyceridemia. Experience of 5 cases]. Rev Med Chil 129:1373-1378

11. Henzen C, Rock M, Schnieper C, Herr K (1999) Heparin and insulin in the treatment of acute hypertriglyceridemia-induced pancreatitis. Schweiz Med Wochenschr 129:1242-1248

12. Näsström B, Olivecrona G, Olivecrona $T$, Stegmayr BG (2001) Lipoprotein lipase during continuous heparin infu- 
sion: tissue stores become partially depleted. J Lab Clin Med 138:206-213

13. Iskandar SB, Olive KE (2004) Plasmapheresis as an adjuvant therapy for hypertriglyceridemia-induced pancreatitis. Am J Med Sci 328:290-294

14. Malmström R, Packard CJ, Watson TDG, Rannikko S, Caslake M, Bedford D, Stewart P, Yki-Järvinen H, Shepherd H, Taskinen M-R (1997) Metabolic basis of hypotriglyceridemic effects of insulin in normal men. Arterioscler Thromb Vasc Biol 17:1454-1464

Intern Emerg Med (2007) 2:70-71

\section{D-dimer testing: advantages and limitations in emergency medicine for managing acute venous thromboembolism}

\section{Imberti}

\author{
D. Imberti (凶) \\ Thrombosis Center \\ Emergency Department \\ Ospedale Civile \\ Via Taverna 49, I-29100 Piacenza, Italy \\ e-mail: d.imberti@ausl.pc.it
}

Received: 5 October 2006 / Accepted in original form: 6 October 2006 / Published online: 31 March 2007

Patients with suspected deep vein thrombosis (DVT) or pulmonary embolism (PE) are frequently admitted to an Emergency Department (ED) for initial evaluation. However, management of patients with suspicion of acute venous thromboembolism (VTE) in this clinical setting can be difficult; in fact symptoms and signs of DVT are non-specific and can be found in a broad spectrum of non-thrombotic disorders. An accurate and timely objective diagnosis is necessary for immediate and correct identification of patients with acute VTE, while avoiding the bleeding risk associated with unnecessary anticoagulant therapy in patients where DVT or PE have been ruled out. The diagnostic approach to patients with suspected VTE includes clinical evaluation, diagnostic imaging and D-dimer testing [1,2].

In a recent issue of Internal and Emergency Medicine, Siragusa [3] exhaustively discussed the currently used assays, clinical indications and limitations of D-dimer testing for managing acute VTE in emergency medicine. Measurement of D-dimer values, a degradation product of cross-linked fibrin, is a simple laboratory test, and has been extensively studied in numerous prospective cohort studies in cases of suspected DVT or PE, showing a high negative predictive value. The author [3] shows that D-dimer testing has sufficient diagnostic accuracy for ruling out acute VTE if used in combination with standardised clinical judgement. The review is important for at least three reasons.

First, many physiological and pathological conditions can increase plasma D-dimer levels (pregnancy, age, trauma, cancer, inflammation and several other clinical conditions); on the other hand D-dimer levels may fail to increase in patients with acute VTE for multiple reasons (impaired fibrinolytic activity, use of heparin or oral anticoagulants, onset of symptoms more than two weeks before blood sampling). For these reasons, D-dimer testing has a high sensitivity but a low specificity in the diagnosis of acute VTE; in clinical practice it may be associated with frequent "false positive" results, but also more rarely with "false negative" findings. However, its use should always be associated with a careful clinical assessment to rule out the presence of acute VTE only in symptomatic patients. Overuse or misuse of the D-dimer screen for VTE may have negative consequences, in terms of a burden both for patients and for healthcare costs; in fact, despite clinical guidelines, inappropriate and unnecessary measurement of D-dimer is a significant clinical problem [4].

Second, commercially available assays are very different for many reasons. Assays differ in the type of antibody used as well as in the type of data provided, as some are qualitative whereas others are quantitative. Moreover, the value of various D-dimer assays is based upon several factors including sensitivity, specificity, cost, time required, reproducibility and complexity. Finally, the diagnostic accuracy of D-dimer relies on the cut-off values, that is the range within which the test has the best sensitivity and specificity, and may be very different comparing one assay to another. Cut-off points are established by the manufacturers, according to the characteristics of D-dimer testing; a wide range of sensitivities and specificities have been reported for different D-dimer assays. Currently, no Ddimer reference standard exists, making interassay correlation poor; moreover, because of variations and differences among different assays, results from one of them cannot be extrapolated to another.

Third, another interesting issue concerning the use of the D-dimer test in emergency medicine is its use when urgent diagnostic imaging for DVT or PE is not immediately available, as for example during a night-time or week-end evaluation. Siragusa and coworkers [5] studied whether a combined approach consisting of pre-test clinical probability, D-dimer testing and low-molecular-weight heparin (LMWH) allowed deferral of objective assessment of DVT or PE for up to $72 \mathrm{~h}$ in 409 patients admitted to ED for suspected VTE. Pending diagnostic confirmation, patients identified with a high probability or with a moderate probability and positive D-dimer test received a protective full dose of LMWH, while the remaining patients were discharged without anticoagulant treatment. The study demonstrates the safety of this approach.

Despite the promise of these findings, there are several points of caution worth noting with respect to the use 